\title{
Sexual Health in COPD: A Systematic Review and Meta-Analysis
}

\author{
Ingeborg Farver-Vestergaard', Yoon Frederiksen ${ }^{2,3}$, Robert Zachariae $\mathbb{D}^{4}$, Sandra Rubio-Rask', \\ Anders Løkke $\mathbb{D}^{1,5}$
}

'Department of Medicine, Vejle Hospital/Lillebaelt Hospital, Vejle, Denmark; ${ }^{2}$ Department of Clinical Medicine, Aarhus University, Aarhus, Denmark; ${ }^{3}$ Unit for Sexology, Aarhus University Hospital Psychiatry, Aarhus, Denmark; ${ }^{4}$ Unit for Psychooncology and Health Psychology, Aarhus University Hospital, Aarhus, Denmark; ${ }^{5}$ Department of Regional Health Research, University of Southern Denmark, Odense, Denmark

Correspondence: Ingeborg Farver-Vestergaard, Department of Medicine, Vejle Hospital, Beriderbakken 4, Vejle, 7100, Denmark, Tel +45 79409832, Email ingeborg.farver@rsyd.dk

\begin{abstract}
COPD has a profound impact on the lives of patients and their partners, but the influence on physical, psychological and social aspects of sexual health has not been reviewed systematically. Searches for studies of the impact of COPD on sexual health were conducted independently by two authors in the databases of PubMed, PsycINFO, Embases, CINAHL, Web of Science, Scopus and The Cochrane Library. English-language quantitative and qualitative studies assessing one or more aspects of sexual health in patients diagnosed with COPD were narratively reviewed and, when possible, subjected to meta-analytic evaluation. A total of 31 studies, including 4 qualitative, were included. Twelve studies assessing erectile dysfunction with the International Index of Erectile Function were subjected to meta-analysis. The pooled prevalence of erectile dysfunction was 74\% (95\% CI: 68-80\%) in a total of 1187 patients with COPD, compared with $56 \%(37-73 \%)$ in 224 age-matched, non-COPD controls. The sexual health outcomes assessed in the remaining studies varied considerably, compromising the comparability of the results. None of the qualitative studies had sexual health as their primary focus. Compared with non-COPD individuals, erectile dysfunction appears to be more prevalent among patients with COPD, but more studies including non-COPD controls are needed to confirm this finding. In addition, the impact of COPD on other physical, psychological and social aspects of sexual health remains unclear due to the lack of comparable assessment methods and study designs.
\end{abstract}

Keywords: sexual problems, chronic obstructive pulmonary disease, psychosocial aspects, gender, quality of life, systematic review

\section{Introduction}

Chronic obstructive pulmonary disease (COPD) is a progressive lung disease, typically diagnosed relatively late in life. ${ }^{1}$ Physical symptoms include breathlessness, cough and excessive sputum production, often accompanied by fatigue, low levels of exercise capacity and psychological symptoms of anxiety and depression. ${ }^{2}$ Hence, the illness has persistent, profound effects on the lives of the patients and their caregivers, including negative impacts on intimate relationships and sexuality. ${ }^{3,4}$

According to the World Health Organization (WHO), sexual health can be described as "a state of physical, emotional, mental and social well-being in relation to sexuality; it is not merely the absence of disease, dysfunction or infirmity". "While, historically, sexual health has been relatively overlooked as a contributing factor to constructs of life satisfaction and quality of life $(\mathrm{QoL}){ }^{6}{ }^{6}$ in recent years, attention has been drawn to aspects of sexual health as strong predictors and correlates of general subjective well-being. ${ }^{7,8}$

Sexual health has been explored widely in somatic diseases such as breast-, colorectal- and testicular cancers, demonstrating direct effects of disease and treatment on hormone regulatory processes, reproductive organs and sexual function. ${ }^{9-11}$ In COPD, on the other hand, changes in sexual health appears to be more indirectly linked to disease symptoms through fear of breathlessness on exertion as well as reductions in functional level and exercise capacity, ${ }^{12}$ but the evidence base on sexual health in COPD is limited. The few existing literature reviews in the area are either non- 
systematic ${ }^{13}$ or focused narrowly on the treatment of physiological deficits such as erectile dysfunction. ${ }^{14}$ Vincent and Singh's narrative review ${ }^{13}$ emphasises the relevance of addressing physical, emotional, relational, social and spiritual factors when assessing the impact of COPD on sexuality, which leads them to advocate for a multidisciplinary approach in future sexual healthcare in COPD. Yet, they build their argumentation mostly on studies of chronic illness or geriatric populations in general, lacking potentially specific aspects of the COPD population. Moreover, the narrative review does not reflect critically on the heterogeneity in the measurement of sexual health aspects. In contrast, the review and metaanalyses by Levack and colleagues ${ }^{14}$ applies a systematic and stringent operationalisation and methodology in their Cochrane-review, but their mechanistic focus on the prevalence of erectile dysfunction and its treatment leaves little insight into 1) other organic factors of sexual health, eg, ejaculation-, orgasmic and pain-related issues, 2) the impact on other biopsychosocial factors of sexual health, which can only partly be explained by organic factors, and 3) the impact of COPD sexual health in female patients. To our knowledge, there have been no later attempts to summarise the empirical literature in the area, and an up-to-date, systematic overview of the literature is therefore warranted.

On this background, the present systematic review aimed to evaluate the impact of COPD on sexual health and to explore the relationship between sexual health and relevant biological, psychological and social variables in this group. Furthermore, we assessed the methodological quality of existing studies with the purpose of critically evaluating the current state of the evidence in the area and identifying implications for clinical practice and future research needs.

\section{Methods}

Prior to the data collection, the review protocol was registered with PROSPERO, The National Institute for Health Research (Reg. ID: CRD42020145161). The methods and results are documented according to the Meta-analysis Of Observational Studies in Epidemiology (MOOSE) Checklist. ${ }^{15}$

\section{Search Strategy and Study Selection}

Literature searches were performed for the period from database inception to January 2021 in the electronic databases of PubMed, PsycINFO, Embases, CINAHL, Web of Science, Scopus and the Cochrane Library, using keywords related to sexual health (sexual OR sexuality OR romantic OR romance OR intimacy OR psychosexual) in combination with keywords related to COPD ("chronic obstructive pulmonary disease" OR "COPD" OR "chronic obstructive lung disease" OR emphysema OR "chronic bronchitis" OR "chronic obstructive respiratory disease" OR "chronic obstructive airway disease"). Relevant additional terms were applied for the individual databases when relevant, eg, using MeSH terms in PubMed and Emtree terms in Embase. Moreover, chain searches were performed by screening the reference lists of included studies. With the purpose of identifying potentially missed studies, supplementary searches were performed using Google Scholar. A trained university librarian was involved in the development of the search strategy and monitored the search procedures.

\section{Inclusion/Exclusion Criteria}

English-language quantitative and qualitative studies were included if they explored aspects of sexual health in patients diagnosed with COPD. If the same study included several different measures of the same variable (eg, an established scale and a non-validated scale), the more well-established and most frequently used scale was selected. Studies were excluded if data from patients with COPD were pooled with data from other populations or chronic conditions. Intervention studies were included if they reported relevant baseline data, but excluded if they reported only change scores from pre- to post-intervention. Abstracts were included if they provided sufficient data.

\section{Study Selection and Data Extraction}

All study screening and selection procedures were performed using the Covidence software (C 2019, v1254 1df2c3cf). Two researchers (IFV and YF) performed the literature search independently. After the elimination of duplicates, the same researchers independently screened all titles and abstracts and excluded ineligible studies. In the second screening round, full-texts of all remaining references were obtained and read in full by both researchers, and they independently 
excluded all ineligible studies and stated inclusion reasons. Relevant data were extracted from the included studies by IFV and cross-checked by YF. Authors of eligible studies were contacted via email to obtain missing data.

\section{Assessment of Methodological Quality}

The methodological quality of the included quantitative studies was assessed using the Appraisal tool for Cross-Sectional Studies (AXIS), ${ }^{16}$ and the Critical Appraisal Skills Programme (CASP) was applied for the methodological assessment of qualitative studies as recommended by the GRADE Working Group. ${ }^{17}$ Two researchers (IFV and YF or SRR and YF) rated the methodological quality of the included studies separately and resolved conflicts when relevant.

\section{Data Synthesis and Analysis}

To enable between-study comparisons, the included studies were grouped according to the specific sexual health instrument used. A random effects meta-analysis was conducted for each group. The impact of COPD on sexual health outcomes was explored by combining the inverse variance weighted mean results for the included studies, thereby taking the precision of each study into consideration. When available for $\geq 5$ studies, demographic, biological, psychological or social variables were explored with meta-regression as possible moderators of sexual health. All analyses were performed using Comprehensive Meta-Analysis, version 3, and various formulas in Microsoft Excel.

Qualitative studies were planned to be subjected to a qualitative data synthesis in accordance with the guidelines of Timulak. ${ }^{18}$

\section{Results}

The study selection process is summarised in the PRISMA flow diagram shown in Figure 1. The initial search yielded 844 unique references out of which 147 articles were read in full during the second screening round. Initially, 23 studies were included, and the supplementary searches yielded an additional 8 eligible studies, resulting in a total of 27 quantitative and 4 qualitative studies published between 1977 and 2021.

\section{Study Characteristics}

The characteristics of the included studies are listed in Table 1, and an overview of the instruments used to measure sexual health outcomes in the included studies can be found in Table 2. An overview of AXIS and CASP study quality ratings can be found in Supplementary Materials, Tables S1 and S2. Twelve studies had sufficient and comparable data for conducting a meta-analysis, ie, had all used the International Index of Erectile Function (IIEF) to measure erectile dysfunction (ED). The instruments used in the remaining studies were too heterogeneous to allow for meta-analytical evaluation.

\section{Erectile Dysfunction}

See Table 2, section A for an overview of studies reporting outcomes on erectile dysfunction. The pooled prevalence of ED in 12 studies of a total of 1187 patients with COPD was 74\% (95\% CI: 68-80\%). The results appeared heterogeneous $(\mathrm{Q}=60.5, \mathrm{p}<0.001)$, with the $I^{2}$ of $81.8 \%$ suggesting that a considerable proportion of the variance can be explained by true differences in prevalence across samples. The $95 \%$ prediction interval indicates that $95 \%$ of similar future studies will yield prevalences between $46.7 \%$ and $90.5 \%$. As seen in Table 3, when attempting to explore the possible sources of heterogeneity with meta-regression, mean age of the sample was associated with increased prevalence of ED, whereas higher mean sample $\mathrm{FEV}^{1}(\%)$ was associated with lower prevalence. The results for the remaining moderators did not reach statistical significance. As only four studies ${ }^{25,31,41,44}$ examined erectile dysfunction in an age-matched non-COPD control group, comparative analysis was considered exploratory. The pooled prevalence in a total of 224 controls was $56 \%$ (95\% CI: $37-73 \%)$. While the prevalence is smaller in controls, as seen when comparing the confidence intervals, the difference between patients with COPD and controls does not reach statistical significance in the available data.

The remainder of studies exploring erectile dysfunction applied other assessment methods, including nocturnal tumescence monitoring and diagnosis codes, and data from these studies could therefore not be included in the metaanalysis. 

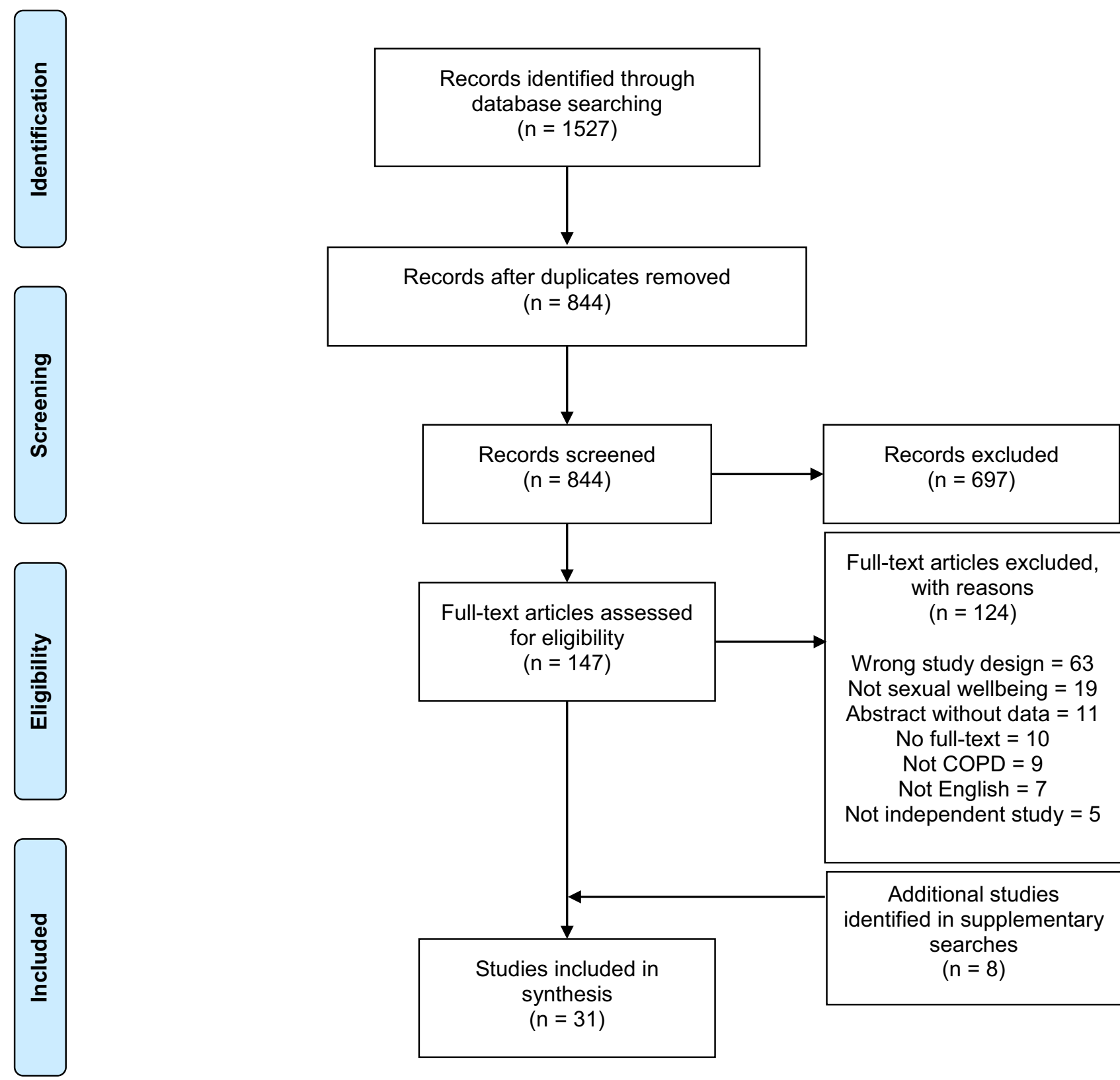

Figure I PRISMA flow diagram of the study selection process.

Notes: Adapted from: Liberati A, Altman D, Tetzlaff J, et al. The PRISMA statement for reporting systematic reviews and meta-analyses of studies that evaluate health care interventions: explanation and elaboration. Journal of Clinical Epidemiology. 2009;62(10)el-e34. ${ }^{74}$ Creative Commons.

\section{Sexual Function and Satisfaction}

See Table 2, section B for an overview of studies reporting outcomes on sexual function and satisfaction. Across studies, limitations in sexual function and satisfaction were reported by $48-82 \%$ and was associated with reduced quality of life, more anxiety and depression symptoms and female gender. Limitations were more common in COPD, compared to healthy samples and other patient populations, eg, heart failure patients.

\section{Illness Impact on Sexual Health}

See Table 2, section C for an overview of studies reporting outcomes on the impact of illness on sexual health. Patients with COPD tended to report greater impact of illness on sexual health compared with patients with heart failure, whereas the impact of asthma and COPD on sexual health were generally more comparable. 
Table I Study Characteristics

\begin{tabular}{|c|c|c|c|c|c|c|c|c|c|}
\hline \multirow[t]{2}{*}{ Author } & \multirow[t]{2}{*}{ Year } & \multirow[t]{2}{*}{ Country } & \multirow[t]{2}{*}{ Study Design } & \multirow[t]{2}{*}{ Study Groups $(n)^{\mathrm{a}}$} & \multicolumn{4}{|c|}{ COPD Sample Characteristics } & \multirow[t]{2}{*}{ Main Findings } \\
\hline & & & & & $\begin{array}{l}\text { Mean } \\
\text { Age } \\
\left(\text { Yrs }^{b}\right)\end{array}$ & $\begin{array}{l}\text { Gender } \\
(\% \\
\text { Female })\end{array}$ & $\begin{array}{l}\text { Lung } \\
\text { Function } \\
\text { (Mean } \\
\text { FEVI \% } \\
\text { Predicted } \\
\text { ) }\end{array}$ & $\begin{array}{l}\text { Partner } \\
\text { Status (\% } \\
\text { Living } \\
\text { with } \\
\left.\text { Partner }{ }^{b}\right)\end{array}$ & \\
\hline $\begin{array}{l}\text { (I) Agle \& } \\
\text { Baum }^{19}\end{array}$ & 1977 & US & $\begin{array}{l}\text { Psychiatric } \\
\text { interviews with } \\
\text { longitudinal } \\
\text { follow-up } \\
\text { (qualitative) }\end{array}$ & $\begin{array}{l}\text { 1) COPD patients } \\
\text { referred to } P R(n=23)\end{array}$ & NR & NR & NR & NR & $\begin{array}{l}\text { Decreased libido and ability to erect was reported by } 19 \\
\text { patients and was attributed to shortness of breath and } \\
\text { easy fatigability, but was not reported to be directly } \\
\text { related to measured physiologic impairment. }\end{array}$ \\
\hline $\begin{array}{l}\text { (2) } \\
\text { Fletcher \& } \\
\text { Martin }^{20}\end{array}$ & 1982 & US & $\begin{array}{l}\text { Prospective } \\
\text { study }\end{array}$ & $\begin{array}{l}\text { I) COPD outpatients } \\
(n=20)\end{array}$ & 56.0 & 0 & NR & 85 & $\begin{array}{l}6 \text { Patients failed to attain an average of one full erection } \\
\text { per night. } 7 \text { participants had ceased sexual activity while } \\
\text { I3 continued sexual activity at } 16 \% \text { of the pre-COPD } \\
\text { sexual activity level. Patients reported interest in engaging } \\
\text { in sexual intercourse to be } 25 \% \text { of pre-COPD level. }\end{array}$ \\
\hline $\begin{array}{l}\text { (3) } \\
\text { Sturesson } \\
\text { \& } \\
\text { Bränholm² }\end{array}$ & 2000 & Sweden & $\begin{array}{l}\text { Cross-sectional } \\
\text { study }\end{array}$ & $\begin{array}{l}\text { 1) COPD patients } \\
\text { receiving LTOT }(n=28) \\
\text { 2) COPD patients } \\
\text { referred to PR }(n=91) \\
\text { 3) Non-COPD control } \\
\text { group }(n=150)\end{array}$ & $\begin{array}{l}\text { LTOT: } 70.0 \\
\text { PR: } 68.0\end{array}$ & $\begin{array}{l}\text { LTOT: 7I } \\
\text { PR: } 45\end{array}$ & NR & NR & $\begin{array}{l}62 \% \text { of the healthy reference group reported being } \\
\text { satisfied with their sex life, which was significantly lower } \\
\text { in the COPD rehabilitation sample ( } 35 \% \text { ) and the COPD } \\
\text { LTOT sample ( } 26 \%) \text {. }\end{array}$ \\
\hline $\begin{array}{l}\text { (4) Ibáñez } \\
\text { et al. }{ }^{4}\end{array}$ & 2001 & Spain & $\begin{array}{l}\text { Cross-sectional } \\
\text { (interview- } \\
\text { based with } \\
\text { quantitative } \\
\text { coding) }\end{array}$ & $\begin{array}{l}\text { I) COPD patients } \\
\text { receiving LTOT }(n=49)\end{array}$ & 68.0 & 0 & $\begin{array}{l}\text { Mean FEVI } \\
\mathrm{mL}=681.3\end{array}$ & 100 & $\begin{array}{l}67.3 \% \text { of the COPD patients showed some type of sexual } \\
\text { problem, out of which } 18 \% \text { manifested as lack of desire, } \\
42 \% \text { as impotence, and } 40 \% \text { as a combination. No } \\
\text { differences in age or lung obstruction were found } \\
\text { between patients who did versus did not report sexual } \\
\text { impairment. }\end{array}$ \\
\hline $\begin{array}{l}(5) \\
\text { O’Neill }\end{array}$ & 2002 & US & $\begin{array}{l}\text { Cross-sectional } \\
\text { interview study } \\
\text { (qualitative) }\end{array}$ & $\begin{array}{l}\text { I) COPD patients } \\
\text { referred to PR (=2I) }\end{array}$ & 67.0 & 100 & NR & 57 & $\begin{array}{l}\text { Patients' perceived consequences of COPD included loss } \\
\text { of intimacy, inability to have sexual relations with their } \\
\text { partners and stigmatisation. }\end{array}$ \\
\hline
\end{tabular}

(Continued) 
Table I (Continued).

\begin{tabular}{|c|c|c|c|c|c|c|c|c|c|}
\hline \multirow[t]{2}{*}{ Author } & \multirow[t]{2}{*}{ Year } & \multirow[t]{2}{*}{ Country } & \multirow[t]{2}{*}{ Study Design } & \multirow[t]{2}{*}{ Study Groups $(n)^{\mathrm{a}}$} & \multicolumn{4}{|c|}{ COPD Sample Characteristics } & \multirow[t]{2}{*}{ Main Findings } \\
\hline & & & & & $\begin{array}{l}\text { Mean } \\
\text { Age } \\
\left(Y_{r s}^{b}\right)\end{array}$ & $\begin{array}{l}\text { Gender } \\
(\% \\
\text { Female })\end{array}$ & $\begin{array}{l}\text { Lung } \\
\text { Function } \\
\text { (Mean } \\
\text { FEVI \% } \\
\text { Predicted }^{\text {b) }}\end{array}$ & $\begin{array}{l}\text { Partner } \\
\text { Status (\% } \\
\text { Living } \\
\text { with } \\
\left.\text { Partner }{ }^{\text {b }}\right)\end{array}$ & \\
\hline $\begin{array}{l}\text { (6) } \\
\text { Svartberg } \\
\text { et al. }\end{array}$ & 2004 & Norway & $\begin{array}{l}\text { RCT (only } \\
\text { baseline data } \\
\text { extracted) }\end{array}$ & $\begin{array}{l}\text { I) COPD outpatients } \\
(\mathrm{n}=29)\end{array}$ & 66.1 & 0 & 42.0 & NR & $\begin{array}{l}\text { IIEF-5 average score at baseline }=14.2 \\
\text { Sexual quality of life average at baseline }=2.3\end{array}$ \\
\hline $\begin{array}{l}\text { (7) } \\
\text { Köseoglu } \\
\text { et al. }{ }^{24}\end{array}$ & 2005 & Turkey & $\begin{array}{l}\text { Cross-sectional } \\
\text { study }\end{array}$ & $\begin{array}{l}\text { I) COPD outpatients } \\
(n=53)\end{array}$ & 63.4 & 0 & NR & 100 & $\begin{array}{l}\text { ED ratio }=75.5 \% \text {. } \\
\text { Low levels of all IIEF domains were associated with longer } \\
\text { COPD duration. Low levels of all IIEF domains, except } \\
\text { sexual desire, were associated decrease more sever } \\
\text { COPD. }\end{array}$ \\
\hline $\begin{array}{l}\text { (8) Karadag } \\
\text { et al. } .^{25}\end{array}$ & 2007 & Turkey & $\begin{array}{l}\text { Cross-sectional } \\
\text { study }\end{array}$ & $\begin{array}{l}\text { I) COPD outpatients } \\
(\mathrm{n}=95)\end{array}$ & 63.5 & 0 & 44.3 & 100 & $\begin{array}{l}\text { ED ratio in } C O P D=87 \% \text { (controls }=83 \% \text { ). Moderate } / \\
\text { severe } E D \text { ratio in } C O P D=57 \% \text { (controls=20\%). Systemic } \\
\text { inflammation }(T N F-\alpha \text { ) level was higher in patients with } \\
\text { moderate } / \text { severe compared with mild } / \text { moderate ED. }\end{array}$ \\
\hline $\begin{array}{l}\text { (9) Kaptein } \\
\text { et } \text { al. }^{26}\end{array}$ & 2008 & $\begin{array}{l}\text { The } \\
\text { Netherlands }\end{array}$ & $\begin{array}{l}\text { Cross-sectional } \\
\text { study }\end{array}$ & $\begin{array}{l}\text { 1) COPD outpatients } \\
(n=25) \\
\text { 2) Non-COPD control } \\
\text { group ( } n=300) \\
\text { 3) Asthma outpatients } \\
(n=30)\end{array}$ & 57.0 & 40 & 58.4 & 100 & $\begin{array}{l}\text { Compared with non-COPD controls, male COPD } \\
\text { patients reported significantly poorer scores on eight out } \\
\text { of nine subscales measuring intimate physical contact, } \\
\text { while female COPD patients reported significantly poorer } \\
\text { scores on one out of nine subscales. General levels of } \\
\text { sexual impairment in asthma was comparable, but male } \\
\text { patients reported less impairment compared to female. }\end{array}$ \\
\hline $\begin{array}{l}\text { (10) } \\
\text { Thomsen \& } \\
\text { Jensen }\end{array}$ & 2009 & Denmark & $\begin{array}{l}\text { Cross-sectional } \\
\text { interview study } \\
\text { (qualitative) }\end{array}$ & $\begin{array}{l}\text { 1) COPD patients after } \\
\text { lung transplantation } \\
(n=10)\end{array}$ & $\begin{array}{l}\text { Range } \\
50-69\end{array}$ & 50 & NR & 40 & $\begin{array}{l}\text { Patients' mentioned resumption of sex life as an important } \\
\text { factor for returning to an ordinary life after lung } \\
\text { transplantation. Their desire to have sex was still present } \\
\text { although problematic due to reduced breathing capacity } \\
\text { and partner's fear that something would go wrong. }\end{array}$ \\
\hline
\end{tabular}




\begin{tabular}{|c|c|c|c|c|c|c|c|c|c|}
\hline $\begin{array}{l}(1 \mathrm{I}) \\
\text { Collins } \\
\text { et al. }\end{array}$ & 2012 & US & $\begin{array}{l}\text { Cross-sectional } \\
\text { study }\end{array}$ & $\begin{array}{l}\text { 1) COPD outpatients } \\
(\mathrm{n}=90)\end{array}$ & 69.0 & 0 & 46.0 & NR & $\begin{array}{l}74 \% \text { had at least one sexual dysfunction, out of these } 72 \% \\
\text { reported erectile dysfunction. Testosterone levels, } \\
\text { significant depressive symptoms and presence of a partner } \\
\text { were independently associated with ED after controlling } \\
\text { for active smoking, comorbidity, obstructive severity, } \\
\text { dyspnea, and medications that could cause ED. }\end{array}$ \\
\hline $\begin{array}{l}(12) \\
\text { Hansen } \\
\text { et al. }{ }^{29}\end{array}$ & 2012 & Denmark & $\begin{array}{l}\text { Cross-sectional } \\
\text { study }\end{array}$ & $\begin{array}{l}\text { 1) Severe COPD } \\
\text { patients }(n=39) \\
\text { 2) Heart failure patients } \\
(\mathrm{n}=22)\end{array}$ & 66.0 & 46 & NR & NR & $\begin{array}{l}\text { A larger proportion of COPD patients reported } \\
\text { troublesome dyspnea during sexual activity compared to } \\
\text { HF patients ( } 44 \% \text { versus } 5 \% \text { ), and that dyspnea was a } \\
\text { limiting factor for sexual activity ( } 56 \% \text { versus } 27 \% \text { ). }\end{array}$ \\
\hline $\begin{array}{l}(13) \\
\text { Uzaslan } \\
\text { et al. }{ }^{30}\end{array}$ & 2012 & $\begin{array}{l}\text { Various, } \\
\text { Middle East }\end{array}$ & $\begin{array}{l}\text { Cross-sectional } \\
\text { epidemiological } \\
\text { study }\end{array}$ & $\begin{array}{l}\text { 1) Patients with a } \\
\text { COPD diagnosis from } \\
\text { the general population } \\
(\mathrm{n}=1392)\end{array}$ & NR & 24.4 & NR & NR & $\begin{array}{l}37.5 \% \text { reported 'some' or 'a lot' of limitation with sexual } \\
\text { intercourse. }\end{array}$ \\
\hline $\begin{array}{l}(14) \\
\text { Kahraman } \\
\text { et al. }\end{array}$ & 2013 & Turkey & $\begin{array}{l}\text { Cross-sectional } \\
\text { study }\end{array}$ & $\begin{array}{l}\text { 1) COPD outpatients } \\
\text { ( } \mathrm{n}=70) \\
\text { 2) Non-COPD control } \\
\text { group }(\mathrm{n}=68)\end{array}$ & 63.34 & 0 & 60.6 & NR & $\begin{array}{l}\text { ED ratio in COPD }=78.6 \% \text { (controls=55.8\%). ED was } \\
\text { associated with lower levels of lung function in COPD } \\
\text { sample, but not in controls. ED was associated with lower } \\
\text { levels of oxygen saturation and higher age in COPD } \\
\text { sample and controls. }\end{array}$ \\
\hline $\begin{array}{l}\text { (15) } \\
\text { Mulhall } \\
\text { et al. }{ }^{32}\end{array}$ & 2013 & US & $\begin{array}{l}\text { Cross-sectional } \\
\text { postal survey }\end{array}$ & $\begin{array}{l}\text { 1) COPD outpatients } \\
(\mathrm{n}=493)\end{array}$ & NR & NR & NR & NR & $\begin{array}{l}\text { Limitations in sexual activity were reported by } 48 \% \text { of the } \\
\text { COPD patients who knew about their diagnosis, } \\
\text { compared to } 23 \% \text { of the COPD who did not know. }\end{array}$ \\
\hline $\begin{array}{l}\text { (16) Panos } \\
\text { et al. }{ }^{33}\end{array}$ & 2013 & US & $\begin{array}{l}\text { Cross-sectional } \\
\text { focus group } \\
\text { study } \\
\text { (qualitative) }\end{array}$ & $\begin{array}{l}\text { I) COPD outpatients } \\
(\mathrm{n}=42)\end{array}$ & 64.6 & 0 & NR & 47 & $\begin{array}{l}\text { Sexual activity was prohibited or severely impeded and } \\
\text { related to marital separation for some individuals. }\end{array}$ \\
\hline $\begin{array}{l}\text { (17) } \\
\text { Theander } \\
\text { et al. }\end{array}$ & 2014 & Sweden & $\begin{array}{l}\text { Cross-sectional } \\
\text { study }\end{array}$ & $\begin{array}{l}\text { 1) Patients with a } \\
\text { COPD diagnosis from } \\
\text { primary health care } \\
\text { registries ( } n=437 \text { ) } \\
\text { 2) Patients with a } \\
\text { chronic heart failure } \\
\text { diagnosis from primary } \\
\text { care registries ( } n=388 \text { ) }\end{array}$ & 70.44 & 54 & NR & 62 & $\begin{array}{l}42 \% \text { of the COPD sample reported problems with sexual } \\
\text { interest or activity, compared to } 33 \% \text { in the chronic heart } \\
\text { failure sample. }\end{array}$ \\
\hline
\end{tabular}


Table I (Continued).

\begin{tabular}{|c|c|c|c|c|c|c|c|c|c|}
\hline \multirow[t]{2}{*}{ Author } & \multirow[t]{2}{*}{ Year } & \multirow[t]{2}{*}{ Country } & \multirow[t]{2}{*}{ Study Design } & \multirow[t]{2}{*}{ Study Groups (n) ${ }^{a}$} & \multicolumn{4}{|c|}{ COPD Sample Characteristics } & \multirow[t]{2}{*}{ Main Findings } \\
\hline & & & & & $\begin{array}{l}\text { Mean } \\
\text { Age } \\
\left(Y_{r s}^{b}\right)\end{array}$ & $\begin{array}{l}\text { Gender } \\
(\% \\
\text { Female) }\end{array}$ & $\begin{array}{l}\text { Lung } \\
\text { Function } \\
\text { (Mean } \\
\text { FEVI \% } \\
\text { Predicted }^{\text {b }} \text { ) }\end{array}$ & $\begin{array}{l}\text { Partner } \\
\text { Status (\% } \\
\text { Living } \\
\text { with } \\
\text { Partner }{ }^{\text {b }} \text { ) }\end{array}$ & \\
\hline $\begin{array}{l}\text { (I8) Abd- } \\
\text { Elsalam } \\
\text { et al. }\end{array}$ & 2015 & Egypt & $\begin{array}{l}\text { Cross-sectional } \\
\text { study }\end{array}$ & $\begin{array}{l}\text { 1) COPD outpatients } \\
(n=86) \\
\text { 2) Non-COPD control } \\
\text { group }(n=86)\end{array}$ & $\begin{array}{l}30 \text { yrs: } \\
14.0 \% \\
31-40 \text { yrs: } \\
41.9 \% \\
41-50 \text { yrs: } \\
37.1 \% \\
51 \text { yrs: } \\
7.0 \%\end{array}$ & 100 & NR & 100 & $\begin{array}{l}\text { Compared with controls, COPD patients scored } \\
\text { significantly lower on the sexual function domains of } \\
\text { desire, arousal, orgasm and sexual satisfaction, and scored } \\
\text { higher on the pain domain. }\end{array}$ \\
\hline $\begin{array}{l}(19) \\
\text { Marques } \\
\text { et al. }^{36}\end{array}$ & 2015 & Portugal & $\begin{array}{l}\text { RCT (only } \\
\text { baseline data } \\
\text { extracted) }\end{array}$ & $\begin{array}{l}\text { I) COPD patients } \\
\text { referred to } P R(n=42)\end{array}$ & 67.4 & 33 & 70.5 & 76 & $\begin{array}{l}\text { Mean scores on a domain scale measuring sexual } \\
\text { relationships was } 3.5 \text {. }\end{array}$ \\
\hline $\begin{array}{l}\text { (20) Shen } \\
\text { et al. }{ }^{37}\end{array}$ & 2015 & Taiwan & $\begin{array}{l}\text { Retrospective } \\
\text { cohort study }\end{array}$ & $\begin{array}{l}\text { 1) Patients with a } \\
\text { COPD diagnosis from } \\
\text { the general population } \\
(n=29,042) \\
\text { 2) Non-COPD control } \\
\text { group from the general } \\
\text { population ( } n=28,886)\end{array}$ & 61.0 & 0 & NR & NR & $\begin{array}{l}\text { After adjusting for age and comorbidity, COPD patients } \\
\text { had an adjusted hazard ratio of } 1.52 \text { ( } 95 \% \mathrm{Cl} 1.30-1.79) \\
\text { for ED in COPD, compared with non-COPD patients. }\end{array}$ \\
\hline $\begin{array}{l}(2 \mathrm{I}) \\
\text { Lauretti } \\
\text { et al. }\end{array}$ & 2016 & Italy & $\begin{array}{l}\text { Cross-sectional } \\
\text { study }\end{array}$ & $\begin{array}{l}\text { I) COPD patients } \\
\text { referred to } P R(n=66)\end{array}$ & 62.4 & 0 & NR & NR & $\begin{array}{l}\text { ED ratio }=83.3 \% \text {. } \\
\text { ED was associated with higher age more severe COPD. }\end{array}$ \\
\hline $\begin{array}{l}(22) \text { Turan } \\
\text { et al. }\end{array}$ & 2016 & Turkey & $\begin{array}{l}\text { Cross-sectional } \\
\text { study }\end{array}$ & $\begin{array}{l}\text { I) COPD outpatients } \\
(n=93)\end{array}$ & 61.4 & 0 & $\begin{array}{l}\text { Mean FEVI } \\
L=I .7 I\end{array}$ & NR & $\begin{array}{l}\text { ED ratio }=67.7 \% \text {. } \\
\text { ED was associated with higher age, more pack-years of } \\
\text { smoking, more dyspnea and lower levels of arterial blood } \\
\text { gases, physical activity QoL. }\end{array}$ \\
\hline $\begin{array}{l}\text { (23) Dias } \\
\text { et al. }\end{array}$ & 2017 & Portugal & $\begin{array}{l}\text { Cross-sectional } \\
\text { study }\end{array}$ & $\begin{array}{l}\text { I) COPD outpatients } \\
(n=67)\end{array}$ & Median $=65$ & 0 & 48.0 & 79 & $\begin{array}{l}\text { ED ratio }=87 \% \\
\text { Higher CAT scores independently associated with } \\
\text { presence of ED (odds ratio=I.123). }\end{array}$ \\
\hline
\end{tabular}




\begin{tabular}{|c|c|c|c|c|c|c|c|c|c|}
\hline $\begin{array}{l}\text { (24) Hasan } \\
\text { et al. }{ }^{41}\end{array}$ & 2017 & Egypt & $\begin{array}{l}\text { Cross-sectional } \\
\text { study }\end{array}$ & $\begin{array}{l}\text { 1) COPD outpatients } \\
(\mathrm{n}=30)\end{array}$ & NR & 0 & NR & NR & $\begin{array}{l}\text { ED ratio= } 76 \% \text { versus } 23 \% \text { in healthy controls. } \\
\text { ED was associated with lower levels of lung function and } \\
\text { oxygen saturation and physical activity and longer COPD } \\
\text { duration. }\end{array}$ \\
\hline $\begin{array}{l}\text { (26) Sinoj } \\
\text { et al. } .^{43}\end{array}$ & 2018 & India & $\begin{array}{l}\text { Cross-sectional } \\
\text { study }\end{array}$ & $\begin{array}{l}\text { I) COPD outpatients } \\
(\mathrm{n}=87)\end{array}$ & 54.1 & 0 & 71.5 & 100 & $\begin{array}{l}\text { ED ratio= }=42.5 \% \\
\text { Presence of } E D \text { was associated with lower levels of lung } \\
\text { function, higher levels of sexual impairment and poorer } \\
\text { marital quality. }\end{array}$ \\
\hline $\begin{array}{l}\text { (27) } \\
\text { Kawshty } \\
\text { et al. }{ }^{44}\end{array}$ & 2019 & Egypt & $\begin{array}{l}\text { Cross-sectional } \\
\text { study }\end{array}$ & $\begin{array}{l}\text { 1) COPD outpatients } \\
(\mathrm{n}=100) \\
\text { 2) Non-COPD control } \\
\text { group }(\mathrm{n}=96)\end{array}$ & 61.5 & 0 & 50.0 & NR & $\begin{array}{l}\text { ED ratio in COPD }=78 \% \text { versus (controls }=58.3 \% \text { ). } \\
\text { Presence of ED was associated with more severe COPD. } \\
\text { Significant, independent predictors of ED were exercise } \\
\text { capacity, testosterone level and lung function. }\end{array}$ \\
\hline $\begin{array}{l}\text { (28) Oh \& } \\
\text { Yoo } 45\end{array}$ & 2019 & Korea & $\begin{array}{l}\text { Longitudinal } \\
\text { cohort study }\end{array}$ & $\begin{array}{l}\text { 1) Patients with COPD } \\
\text { diagnosis from a } \\
\text { hospital-based cohort } \\
(n=185)\end{array}$ & 65.4 & 0 & 59.9 & NR & $\begin{array}{l}\text { Erectile function in total sample improved in the first year } \\
\text { after baseline and then deteriorated. } \\
\text { Older age, lower economic status and poorer subjective } \\
\text { health status affected the progression of erectile function. }\end{array}$ \\
\hline $\begin{array}{l}\text { (30) } \\
\text { Zysman } \\
\text { et al. }{ }^{47}\end{array}$ & 2020 & France & $\begin{array}{l}\text { Cross-sectional } \\
\text { survey }\end{array}$ & $\begin{array}{l}\text { 1) Patients with self- } \\
\text { reported COPD } \\
(n=75 I)\end{array}$ & 61.0 & 51 & $\begin{array}{l}\text { FEV } 1 \% \text { pred } \\
\leq 30=23.0\end{array}$ & 62 & $\begin{array}{l}68 \% \text { Reported 'low/absent' sexual appetite, } 60 \% \text { reported } \\
\text { 'low/absent' sexual desire, } 60 \% \text { of male participants } \\
\text { reported erectile dysfunction. Low/absent sexual appetite } \\
\text { or desire was associated with higher levels of anxiety and } \\
\text { depression. }\end{array}$ \\
\hline $\begin{array}{l}\text { (3l) Kamal } \\
\text { et al. }{ }^{48}\end{array}$ & 2021 & Egypt & $\begin{array}{l}\text { Cross-sectional } \\
\text { study }\end{array}$ & $\begin{array}{l}\text { I) COPD outpatients } \\
(n=100)\end{array}$ & 56.3 & 0 & NR & NR & $\begin{array}{l}\text { ED ratio in COPD }=71 \% \\
\text { Mean levels of erectile function were significantly higher in } \\
\text { controls, compared to COPD. } \\
\text { Higher levels erectile function were associated with } \\
\text { higher levels of testosterone. }\end{array}$ \\
\hline
\end{tabular}

Notes: ${ }^{2}$ Non-COPD control samples are age- and gender-matched; bUnless reported otherwise.

Abbreviations: COPD, chronic obstructive pulmonary disease; ED, erectile dysfunction; FEVI, forced expiratory volume in the first second; IIEF, International Index of Erectile Function; NR, not reported; PR, pulmonary rehabilitation: $\mathrm{RCT}$, randomised controlled trial. 
Table 2 Overview of Instruments Applied to Measure Sexual Health in the Included Studies

\begin{tabular}{|c|c|c|c|c|c|c|c|}
\hline Outcome & $\begin{array}{l}\text { Measurement } \\
\text { Instrument }\end{array}$ & $\begin{array}{l}\text { Sexual health } \\
\text { Outcome }\end{array}$ & Subdomains & $\begin{array}{l}\text { Number } \\
\text { of Items }\end{array}$ & $\begin{array}{l}\text { Total Score } \\
\text { Range }\end{array}$ & Interpretation & $\begin{array}{l}\text { Applied in } \\
\text { Which } \\
\text { Studies }\end{array}$ \\
\hline \multirow[t]{4}{*}{$\begin{array}{l}\text { A) Erectile } \\
\text { dysfunction }\end{array}$} & $\begin{array}{l}\text { International Index of } \\
\text { Erectile Function-5 } \\
(\text { IIEF-5) }\end{array}$ & $\begin{array}{l}\text { Presence and } \\
\text { severity of erectile } \\
\text { dysfunction }\end{array}$ & Erectile function & 5 & $5-25$ & $\begin{array}{l}\text { Cutoff score for } \\
\text { presence of erectile } \\
\text { dysfunction }=21 \\
\text { Higher scores indicating } \\
\text { higher levels of erectile } \\
\text { function }\end{array}$ & $\begin{array}{l}{[23,25,31,39-} \\
42,44,45,48]\end{array}$ \\
\hline & $\begin{array}{l}\text { International Index of } \\
\text { Erectile Function - full } \\
\text { scale }^{50}\end{array}$ & $\begin{array}{l}\text { Erectile function and } \\
\text { satisfaction }\end{array}$ & $\begin{array}{l}\text { Erectile function; orgasmic function; sexual desire; } \\
\text { intercourse satisfaction; overall satisfaction }\end{array}$ & 15 & $4-55$ & $\begin{array}{l}\text { Higher scores indicating } \\
\text { higher levels of erectile } \\
\text { function }\end{array}$ & {$[24,38,43]$} \\
\hline & $\begin{array}{l}\text { Tumescence } \\
\text { monitor }^{51-53}\end{array}$ & $\begin{array}{l}\text { Erectile function } \\
\text { measured by } \\
\text { circumferential } \\
\text { change of the penis } \\
\text { during sleep }\end{array}$ & Erection & I & Yes/no & - & [20] \\
\hline & Diagnosis codes & $\begin{array}{l}\text { Erectile dysfunction } \\
\text { (ICD-9-CM codes } \\
302.72,607.84)\end{array}$ & - & I & Yes/no & - & [37] \\
\hline
\end{tabular}




\begin{tabular}{|c|c|c|c|c|c|c|c|}
\hline \multirow[t]{7}{*}{$\begin{array}{l}\text { B) Sexual } \\
\text { function } \\
\text { and } \\
\text { satisfaction }\end{array}$} & $\begin{array}{l}\text { Respiratory } \\
\text { Experiences with } \\
\text { Sexuality Profile } \\
(\text { RESP) })^{54}\end{array}$ & $\begin{array}{l}\text { Impact of } \\
\text { respiratory } \\
\text { symptoms on } \\
\text { sexuality }\end{array}$ & $\begin{array}{l}\text { "During sexual activity I experience breathing } \\
\text { difficulties"; } \\
\text { "Because of my breathing difficulties I am not capable to } \\
\text { be as sexually active as I would like to be"; } \\
\text { "I talk with my partner about the consequences that my } \\
\text { breathing difficulties have on our sexual activity"; } \\
\text { "I talk with my physician about the consequences that my } \\
\text { breathing difficulties have on my sexual activity" }\end{array}$ & 4 & $\begin{array}{l}\text { Items are not } \\
\text { summed but } \\
\text { reflect the } \\
\text { responses of } \\
\text { individual items: } \\
\text { I: 'Often' } \\
\text { 2: 'Frequently' } \\
\text { 3: 'Sometimes' } \\
\text { 4: 'Never' }\end{array}$ & $\begin{array}{l}\text { Items I and 2: 'Often' is } \\
\text { negative and 'never' is } \\
\text { positive } \\
\text { Items } 3 \text { and 4: 'Often' is } \\
\text { positive and 'never' is } \\
\text { negative }\end{array}$ & {$[26,29,40]$} \\
\hline & $\begin{array}{l}\text { Male Sexual Quotient } \\
(\text { MSQ })^{55}\end{array}$ & $\begin{array}{l}\text { Male sexual function } \\
\text { and satisfaction }\end{array}$ & $\begin{array}{l}\text { Desire; confidence; foreplay quality; partner satisfaction; } \\
\text { erection quality; ejaculatory control; ability to achieve } \\
\text { orgasm; intercourse satisfaction }\end{array}$ & 10 & $0-100$ & $\begin{array}{l}\text { Higher scores indicating } \\
\text { higher levels of sexual } \\
\text { function and satisfaction }\end{array}$ & [46] \\
\hline & $\begin{array}{l}\text { Female Sexual } \\
\text { Quotient (FSQ) })^{56}\end{array}$ & $\begin{array}{l}\text { Female sexual } \\
\text { function and } \\
\text { satisfaction }\end{array}$ & Not reported & 10 & $0-100$ & $\begin{array}{l}\text { Higher scores indicating } \\
\text { higher levels of sexual } \\
\text { function and satisfaction }\end{array}$ & [46] \\
\hline & $\begin{array}{l}\text { Modified Global Study } \\
\text { of Sexual Attitudes } \\
\text { and Behaviors } \\
\text { (GSSAB) } \\
\text { Questionnaire }^{57}\end{array}$ & $\begin{array}{l}\text { Subjective sexual } \\
\text { well-being }\end{array}$ & $\begin{array}{l}\text { Emotional satisfaction with sexual relationships; Physical } \\
\text { satisfaction with sexual relationships; Satisfaction with } \\
\text { sexual function; Importance of sex in overall life }\end{array}$ & NR & NR & NR & [28] \\
\hline & $\begin{array}{l}\text { Arizona Sexual } \\
\text { Experience Scale } \\
(\text { ASEX) })^{58}\end{array}$ & $\begin{array}{l}\text { Sexual function and } \\
\text { satisfaction }\end{array}$ & $\begin{array}{l}\text { Drive; arousal; penile erection/vaginal lubrication; ability } \\
\text { to reach orgasm; satisfaction from orgasm }\end{array}$ & 5 & $5-30$ & $\begin{array}{l}\text { Higher scores indicating } \\
\text { lower levels of sexual } \\
\text { function and satisfaction }\end{array}$ & [47] \\
\hline & $\begin{array}{l}\text { Female Sexual } \\
\text { Function Index } \\
(\text { FSFI) })^{59}\end{array}$ & $\begin{array}{l}\text { Female sexual } \\
\text { function and } \\
\text { satisfaction }\end{array}$ & Desire; arousal; lubrication, orgasm, satisfaction, pain & 19 & $2-36$ & $\begin{array}{l}\text { Higher scores indicating } \\
\text { higher levels of sexual } \\
\text { function and satisfaction }\end{array}$ & [35] \\
\hline & $\begin{array}{l}\text { Single item from the } \\
\text { Memorial Symptom } \\
\text { Assessment Scale }^{60}\end{array}$ & $\begin{array}{l}\text { "Problems with } \\
\text { sexual interest or } \\
\text { activity" }\end{array}$ & - & I & Yes/no & - & [34] \\
\hline
\end{tabular}


Table 2 (Continued).

\begin{tabular}{|c|c|c|c|c|c|c|c|}
\hline Outcome & $\begin{array}{l}\text { Measurement } \\
\text { Instrument }\end{array}$ & $\begin{array}{l}\text { Sexual health } \\
\text { Outcome }\end{array}$ & Subdomains & $\begin{array}{l}\text { Number } \\
\text { of Items }\end{array}$ & $\begin{array}{l}\text { Total Score } \\
\text { Range }\end{array}$ & Interpretation & $\begin{array}{l}\text { Applied in } \\
\text { Which } \\
\text { Studies }\end{array}$ \\
\hline & $\begin{array}{l}\text { Items formulated by } \\
\text { authors (non- } \\
\text { validated) }\end{array}$ & $\begin{array}{l}\text { Satisfaction with } \\
\text { sexuality } \\
\text { Sexual quality of life } \\
\text { (not specified) } \\
\text { Limitations in sexual } \\
\text { activity }\end{array}$ & - & $\begin{array}{l}1 \\
1 \\
1\end{array}$ & $\begin{array}{l}1-6 \\
0-6 \\
\text { Yes/no }\end{array}$ & $\begin{array}{l}\text { Higher score = higher } \\
\text { satisfaction } \\
\text { Higher score = higher } \\
\text { quality- }\end{array}$ & {$[21,23,32]$} \\
\hline \multirow[t]{5}{*}{$\begin{array}{l}\text { C) Illness } \\
\text { impact on } \\
\text { sexual } \\
\text { health }\end{array}$} & $\begin{array}{l}\text { Intimate Physical } \\
\text { Contacts Scale } \\
\text { (IPCS) }^{61}\end{array}$ & $\begin{array}{l}\text { Illness impact on } \\
\text { intimate physical } \\
\text { contacts }\end{array}$ & $\begin{array}{l}\text { Physical problems influencing sexual desire; frequency of } \\
\text { excitement; frequency of intimacy; appreciation of } \\
\text { intimacy; appreciation of excitement; low self-esteem } \\
\text { interfering with sexuality; low esteem for partner } \\
\text { interfering with sexuality; general assessment of partner; } \\
\text { general sexual satisfaction }\end{array}$ & 9 & NR & $\begin{array}{l}\text { Individual interpretation } \\
\text { for each item }\end{array}$ & [26] \\
\hline & $\begin{array}{l}\text { Psychosocial } \\
\text { Adjustment to Illness } \\
\text { Scale (PAIS) Sexual } \\
\text { relationships } \\
\text { subdomain }^{62}\end{array}$ & $\begin{array}{l}\text { Illness impact on } \\
\text { sexual functioning or } \\
\text { relationships }\end{array}$ & The scale is a subscale of the PAIS & 6 & $0-18$ & $\begin{array}{l}\text { Higher scores indicating } \\
\text { higher levels of illness } \\
\text { impact on sexual } \\
\text { functioning or } \\
\text { relationships }\end{array}$ & [36] \\
\hline & $\begin{array}{l}\text { Relationship and } \\
\text { Sexuality scale [NR] }\end{array}$ & $\begin{array}{l}\text { Illness and treatment } \\
\text { impact on sexuality } \\
\text { and relationships }\end{array}$ & Sexual function; sexual frequency; sexual fear & 10 & $0-36$ & $\begin{array}{l}\text { Higher scores indicating } \\
\text { higher levels of } \\
\text { dysfunction }\end{array}$ & [43] \\
\hline & $\begin{array}{l}\text { Coded from semi- } \\
\text { structured interviews } \\
\text { (non-validated) }\end{array}$ & $\begin{array}{l}\text { Illness impact on } \\
\text { sexuality }\end{array}$ & Impotence; lack of desire & 2 & Yes/no & - & [4] \\
\hline & $\begin{array}{l}\text { Items formulated by } \\
\text { authors (non- } \\
\text { validated) }\end{array}$ & $\begin{array}{l}\text { Impact of COPD on } \\
\text { sexual intercourse }\end{array}$ & - & I & NR & NR & [30] \\
\hline
\end{tabular}

Abbreviations: COPD, chronic obstructive pulonary disease; NR, not reported. 
Table 3 Exploratory Meta-Regression: Moderators of the Pooled Prevalence of Erectile Dysfunction in Samples of COPD Patients

\begin{tabular}{|l|l|l|l|}
\hline Moderator Variable & $\mathbf{K}^{\mathbf{a}}$ & Slope $(\mathbf{9 5 \%} \mathbf{C l})^{\mathbf{b}}$ & $\mathbf{P}^{\mathbf{c}}$ \\
\hline Mean age of sample (years) & $\mathrm{II}$ & $0.095(0.028-0.162)$ & $\mathbf{0 . 0 0 5}$ \\
Mean BMI in sample & 6 & $-0.057(-0.322-0.208)$ & 0.673 \\
Mean pack-years in sample & 5 & $0.035(-0.033-0.102)$ & 0.314 \\
$\mathrm{COPD}$ duration (months) & 6 & $0.006(-0.005-0.017)$ & 0.277 \\
Mean sample $\mathrm{PaO}_{2}$ & 6 & $-0.022(-0.052-0.008)$ & 0.147 \\
Mean sample $\mathrm{PaCO}_{2}$ & 6 & $0.055(-0.048-0.159)$ & 0.296 \\
Mean sample FEVI (\%) & 7 & $-0.015(-0.088-0.03 \mathrm{I})$ & $<0.00 \mathrm{I}$ \\
IIEF-5 vs IIEF (referent) & $\mathrm{II}$ & $0.450(-0.305-1.205)$ & 0.242 \\
\hline
\end{tabular}

Notes: a Number of studies in the analysis; ${ }^{b}$ Mixed effects regression, unrestricted maximum likelihood, calculated for $\mathrm{K} \geq 5$; ${ }^{\mathrm{c} S t a t i s t i c a l l y ~ s i g n i f i c a n t ~ v a l u e s ~}<0.05$ are presented in bold.

In the qualitative studies, sexual impairment was attributed to shortness of breath and easy fatigability. Moreover, qualitative data pointed to the perceived impact of COPD on relational factors, including loss of intimacy, stigmatisation and partner's fear that something would go wrong. Nevertheless, patients described that their desire to have sex was still present.

\section{Discussion}

The present study systematically reviewed existing studies of sexual health in COPD, applying the term 'sexual health' as an umbrella term for aspects of sexuality instead of focusing solely on specific sexual functions, eg, erectile dysfunction. A total of 27 quantitative studies and 4 qualitative studies was included. Studies were generally heterogeneous in terms of recruitment settings, measurement instruments as well as study designs.

Despite overall heterogeneity, the majority of the included quantitative studies focused on biological deficits related to sexual function, such as erectile dysfunction (ED), in male patients with COPD. Due to large variation in measurement instruments applied across the included quantitative studies, it was only possible to calculate a quantitative, summarised estimate of COPD impact on the outcome of ED as assessed with the International Index of Erectile Dysfunction (IIEF).

\section{Erectile Dysfunction}

Meta-analysis results showed that three out of four patients with COPD met the criteria for a diagnosis of ED, which represented a higher - albeit not statistically significant - proportion than in age-matched, non-COPD control samples. The lack of statistical significance of this finding may be due to lack of power, with only 224 participants being included in the pooled control sample. In a recently published meta-analysis, Luo et $\mathrm{al}^{63}$ directly compared the risk of developing ED in patients with COPD and non-COPD, age-matched controls. They found that the risk of developing moderate or severe ED was significantly higher in COPD, compared to the age-matched general population. However, the risk was not increased for ED in general.

ED results in the present study were heterogeneous, and exploratory analyses showed that higher mean age was associated with increased prevalence of ED. This trend was similar in an umbrella meta-analysis of the global prevalence of ED, eg, prevalences of $\sim 25 \%$ at age 30-39 and $\sim 80$ at age $60-69 .{ }^{64}$ Furthermore, results of the present study showed that better lung function was associated with lower ED prevalence. There may be numerous explanatory factors for this finding. For example, the vast majority of patients with COPD are current or former smokers, and higher levels of both past and current cigarette smoking have been shown to be associated with increased ED risk in the general population. ${ }^{64}$ However, results of the moderating effects of smoking, body mass index (BMI), COPD duration, partial pressure of oxygen $\left(\mathrm{PaO}_{2}\right)$, and partial pressure of carbon dioxide $\left(\mathrm{PaCO}_{2}\right)$ did not reach statistical significance. This could, however, be caused by power issues, and should therefore be interpreted with caution. 


\section{Sexual Function and Satisfaction}

In the present review, studies that applied instruments assessing sexual function and satisfaction generally reported low levels of function and satisfaction among patients with COPD. Sexual function and satisfaction was shown to be associated with higher levels of anxiety and depression. ${ }^{46,47}$ However, the causality of this association is unclear, since psychological symptoms could be both a reason and a consequence of poor sexual function. ${ }^{65}$ Poor sexual function was more frequently reported among women, which can generally not be explained by gender differences in psychological symptoms. However, since the majority of studies of sexual health in COPD focus on sexual function among male patients, it is not possible to draw reliable conclusions on gender difference based on existing data, and more studies applying non-gender specific assessment instruments are needed.

\section{Illness Impact on Sexual Health}

In the present review, a number of the included studies applied measurement instruments that directly assess illness impact on sexual health. The included studies indicated that sexual health of patients with COPD is compromised by respiratory symptoms to a similar extent as in patients with asthma, whereas the impact of respiratory symptoms on sexual health is more profound in patients with COPD, compared to patients with heart failure. Whether or not these results can be explained by differences in respiratory symptoms across different patient groups is currently not clear, and more research is needed to confirm the disease-specific mechanisms of sexual health in COPD.

The included qualitative studies emphasised the influence of relational factors for understanding the impact of COPD on sexual health, where loss of intimacy, patients' perceived inability to have sexual relations with their partners and partners' fear of patients' breathing problems during sexual activities, was highlighted. It is highly relevant to consider relational factors, eg, dyadic coping, ${ }^{66}$ in order to understand interactions, activities and cognitions related to sexuality and sexual health. In studies of cancer populations, aspects of sexual health can be both a predictor and outcome of dyadic coping. ${ }^{67}$ In a study of dyadic coping in COPD,${ }^{68}$ patients as well as partners reported low levels of satisfaction with dyadic coping, compared to a norm group. According to the authors, this finding can be linked with the limited sexual activity in couples with one partner suffering from COPD. A longitudinal study of dyadic coping in COPD ${ }^{69}$ concludes that, as the illness progresses, it is important that both partners communicate about stress and provide appropriate practical and emotional support to maintain quality of life. However, neither studies explore the role of sexual health in dyadic coping, which should be a priority in future studies, as dyadic coping with COPD may differ from coping with malignant diseases or diseases with different illness and treatment trajectories.

\section{Methodological Quality}

With regard to the methodological quality of the included studies, the systematic quality ratings of the present review generally point to four areas with potential for improvement in future studies: First, the majority of included studies failed to describe their approach to sample size justification. While sample size justification is less commonly performed and reported in observational studies, compared to RCTs, a systematic consideration of the number of participants required to estimate prevalence levels in a given population is important with the purpose of increasing the precision of estimates, and sample size justification should therefore be described in future studies. Second, only few of the included studies systematically addressed and categorised non-responders, which compromises the external validity of the study results. Future studies should therefore pay careful attention to the systematic registration and characterisation of nonresponders. Third, and related to the second point, concerns about non-response bias was raised due to limited response rates, and a lack of description of reasons for non-response, in the majority of studies. Fourth, the majority of included studies failed to report study limitations, which challenges the reader's interpretation and applicability of study results. Concerning the included qualitative studies, the methodological quality of three out of four included studies was relatively high. It should be noted, however, that is it questionable whether the remaining study ${ }^{19}$ could be classified as a qualitative study at all. 


\section{Strengths and Limitations}

The present review is the first attempt to systematically review and summarise existing quantitative and qualitative studies on sexual health in COPD. Among its strengths are the adherence to existing systematic guidelines for preregistration, literature search and methodological quality rating, with two reviewers performing procedures independently with the purpose of reducing the risk of bias in selection and reporting. While meta-analysis has been performed to obtain a more precise prevalence estimate, the review also takes a broader approach to sexual health. Historically, studies of erectile dysfunction have dominated the field, and the present study is the first systematic attempt to set a more comprehensive agenda for exploring sexual health in COPD.

A number of limitations of the present review should also be mentioned. First, meta-analytic evaluation and qualitative result syntheses of the including studies could not be performed for all outcomes due to heterogeneity in study objectives and measurement instruments across studies. Limiting the scope to one primary outcome in future systematic reviews may result in a more homogenous evidence base, but might also result in an oversimplified approach to sexual health. Second, the tools applied for methodological quality ratings of quantitative and qualitative studies, respectively, are not developed congruently, and does therefore assess diverse aspects of methodological quality (eg, AXIS focus more extensively on reporting, compared to CASP that focus relatively more on study design). The development of congruent quality rating systems for cross-sectional quantitative and qualitative studies could be relevant for future research. Third, while the present study aimed to evaluate the impact of COPD on sexual health and to explore the relationship between sexual health and relevant biological, psychological and social variables in patients with COPD, treatment methods to improve sexual health in this group was not within the scope. When sufficient knowledge on the prevalence and underlying mechanisms of sexual health in COPD has been obtained, studies developing and testing treatment approaches are needed. Fourth, studies included in the present review were conducted in various settings, ie, outpatient $(\mathrm{K}=9)$, rehabilitation $(\mathrm{K}=3)$, transplantation/long-term oxygen treatment $(\mathrm{K}=4)$ or epidemiological $(\mathrm{K}=3)$, resulting in relatively diverse samples. The considerable heterogeneity in outcomes and study design within study settings makes direct comparison of study results across settings difficult and greater uniformity in settings, outcomes and designs are warranted in future studies. And fifth, a vast number of the included studies were performed in Eastern, Middle Eastern or North African regions of the world, eg, Egypt, ${ }^{35,41,44,48}$ India, ${ }^{43}$ Korea, ${ }^{45}$ Taiwan, ${ }^{37}$ Turkey, ${ }^{24,25,31,39}$ Pakistan, ${ }^{42}$ mixed. ${ }^{30}$ Sexual norms in these areas may differ from Western and Nordic regions, thereby questioning the cultural generalisability of the present results.

\section{Clinical Implications}

Based on results from the meta-analysis of the present study, the majority of male patients with COPD suffer from comorbid ED, potentially influencing their sexual health, partnership and quality of life. Meanwhile, studies on communication needs in COPD indicate that in more than $60 \%$ of the patients with COPD, sexual issues have not been addressed in healthcare consultations, despite a patient-reported need for information. ${ }^{70}$ Apparently, patients are increasingly motivated to achieve information about sexual and relational issues during the first five years after their COPD diagnosis, and especially male patients lack information on these issues. ${ }^{70}$ This highlights the need for a systematic approach to sexual health screening, which should be initiated shortly after diagnosis and maintained throughout the illness trajectory as age increases and lung function decreases. Since most studies of sexual health in COPD have focused on ED, results are currently more robust for this outcome, compared to outcomes of general sexual function, sexual satisfaction and illness impact on sexual health. However, this points to the current state of the evidence in the area, and not necessarily to which outcomes of sexual health have most impact on patients' health status and quality of life. When screening for comorbid diagnosis of ED, clinicians should be careful not to overlook other important factors of sexual health by applying existing, validated questionnaires (see Table 2) - especially when it comes to female patients.

Nonetheless, interpersonal barriers, social stigma and myths about sexuality, eg, "older people with chronic illness automatically looses interest in sex", can hinder the initiation of screening and communication about sexual health. ${ }^{71}$ If designed properly, counselling guidelines for the clinician ${ }^{71}$ and informational leaflets or other types of patient material 
can serve as tools to support communication about sexual issues, as long as they do not end up being used "mechanically" or as a substitute to a clinical conversation. ${ }^{72}$ When sexual or relational issues have been identified in the clinic, relevant management of such issues is needed. Research about medical treatment of ED is limited, ${ }^{14}$ and most likely, sexual health management in COPD demands a holistic, rehabilitative approach, preferably including the partner when there is one. Such an approach could be composed by relevant components, eg, medical management, lifestyle and behaviour change interventions, psychological and psychosexual counselling, and/or dyadic coping interventions. ${ }^{66,68,73}$

\section{Conclusions}

The results of the present study indicate that COPD has a negative impact on sexual health. The majority of included studies focus on organic deficits in male patients, ie, erectile dysfunction (ED), and it is estimated that three out of four patients with COPD are living with erectile dysfunction, which is a larger proportion than in age-matched, non-COPD individuals. However, while sexual health cannot be reduced to organic deficits such as erectile dysfunction, included studies exploring physical, psychological and social aspects of sexual health are limited in number and apply heterogeneous outcomes measures and design, which challenges the comparability of their results. Also, there are currently very few qualitative studies of sexual health in COPD, and none of them has sexual health as the primary study focus. Moreover, the field appears to be dominated by methodological challenges, especially concerning sample size justification, analysis of non-responders and dropouts, and authors' consideration of study limitations. Taken together, the state of the evidence base in COPD and sexual health can be considered relatively poor, compared to sexual health research in other patient populations, eg, cancer. In the future, studies should consider 1) including non-COPD control groups with the purpose of exploring COPD-specificity of results; 2) applying existing, validated measures of sexual health that are applicable in both genders; 3) not limiting outcomes to organic deficits, such as erectile dysfunction; and 4) exploring the subjective experience of COPD impact on sexual health in patients and partners with the purpose of identifying COPDspecific areas that may have been overlooked so far.

\section{Acknowledgment}

A special thanks to Gina Bay, Aarhus University Library for assistance in relation to literature search procedures.

\section{Funding}

This work was supported by the Danish Lung Association Research Fund and Jens Ove Jacobsen's Foundation.

\section{Disclosure}

Dr Ingeborg Farver-Vestergaard reports personal fees from Boehringer Ingelheim, Novartis, and Roche, outside the submitted work. The authors report no other potential conflicts of interest for this work.

\section{References}

1. Yayan J, Rasche K. Asthma and COPD: similarities and differences in the pathophysiology, diagnosis and therapy. Adv Exp Med Biol. 2016;910:3138.

2. GOLD. Global strategy for the diagnosis, management, and prevention of chronic obstructive pulmonary disease; 2017.

3. Gatti V, Banfi P, Centanni S, et al. Enlightening chronic obstructive pulmonary disease through patients' and caregivers' narratives. Int J COPD. 2018;13:3095-3105. doi:10.2147/COPD.S172214

4. Ibáñez M, Aguilar JJ, Maderal MA, et al. Sexuality in chronic respiratory failure: coincidences and divergences between patient and primary caregiver. Respir Med. 2001;95(12):975-979. doi:10.1053/rmed.2001.1192

5. World Health Organization. Sexual and reproductive health and research including the special programme HRP. Available from:https://www.who.int/ teams/sexual-and-reproductive-health-and-research/key-areas-of-work/sexual-health/defining-sexual-health. Accessed October 15, 2021.

6. Hooghe M. Is sexual well-being part of subjective well-being? An empirical analysis of Belgian (Flemish) survey data using an extended well-being scale. J Sex Res. 2012;49(2-3):264-273. doi:10.1080/00224499.2010.551791

7. Stephenson KR, Meston CM. The conditional importance of sex: exploring the association between sexual well-being and life satisfaction. $J$ Sex Marital Ther. 2015;41(1):25-38. doi:10.1080/0092623X.2013.811450

8. Davison SL, Bell RJ, LaChina M, Holden SL, Davis SR. The relationship between self-reported sexual satisfaction and general well-being in women. J Sex Med. 2009;6(10):2690-2697. doi:10.1111/j.1743-6109.2009.01406.x

9. Jonker-Pool G, Van de Wiel HBM, Hoekstra HJ, et al. Sexual functioning after treatment for testicular cancer-review and meta-analysis of 36 empirical studies between 1975-2000. Arch Sex Behav. 2001;30(1):55-74. doi:10.1023/A:1026468707362 
10. Traa MJ, De Vries J, Roukema JA, Den Oudsten BL. Sexual (dys) function and the quality of sexual life in patients with colorectal cancer: a systematic review. Ann Oncol. 2012;23(1):19-27. doi:10.1093/annonc/mdr133

11. Emilee G, Ussher JM, Perz J. Sexuality after breast cancer: a review. Maturitas. 2010;66(4):397-407. doi:10.1016/j.maturitas.2010.03.027

12. Levack W. Sexual wellbeing for people with chronic obstructive pulmonary disease: relevance and roles for physiotherapy. New Zeal J Physiother. 2014;42(3):154-161

13. Vincent EE, Singh SJ. Addressing the sexual health of patients with COPD: the needs of the patient and implications for health professionals. Chron Respir Dis. 2007;4:111-115. doi:10.1177/1479972306076105

14. Levack William MM, Poot B, Weatherall M, Travers J. Interventions for sexual dysfunction in people with chronic obstructive pulmonary disease (COPD). Cochrane Database Syst Rev. 2014;(12). doi: 10.1002/14651858.CD011442

15. Stroup DF, Berlin JA, Morton SC, et al. Meta-analysis of observational studies in epidemiology: a proposal for reporting. $J$ Am Med Assoc. 2000;283(15):2008-2012. doi:10.1001/jama.283.15.2008

16. Downes MJ, Brennan ML, Williams HC, Dean RS. Development of a critical appraisal tool to assess the quality of cross-sectional studies (AXIS). BMJ Open. 2016;6(12):1-7. doi:10.1136/bmjopen-2016-011458

17. Munthe-Kaas H, Bohren MA, Glenton C, et al. Applying GRADE-CERQual to qualitative evidence synthesis findings-paper 3: how to assess methodological limitations. Implement Sci. 2018;13:23-32. doi:10.1186/s13012-017-0690-9

18. Timulak L. Meta-analysis of qualitative studies: a tool for reviewing qualitative research findings in psychotherapy. Psychother Res. 2009;19:591600. doi:10.1080/10503300802477989

19. Agle DP, Baum GL. Psychological aspects of chronic obstructive pulmonary disease. Med Clin North Am. 1977;61(4):749-758. doi:10.1016/ S0025-7125(16)31294-9

20. Fletcher EC, Martin RJ. Sexual dysfunction and erectile impotence in chronic obstructive pulmonary disease. Chest. 1982;81(4):413-421. doi:10.1378/chest.81.4.413

21. Sturesson M, Branholm I-B. Life satisfaction in subjects with chronic obstructive pulmonary disease. Work. 2000;14(2):77-82.

22. O’Neill ES. Illness representations and coping of women with chronic obstructive pulmonary disease: a pilot study. Hear Lung J Acute Crit Care. 2002;31(4):295-302. doi:10.1067/mhl.2002.123712

23. Svartberg J, Aasebo U, Hjalmarsen A, Sundsfjord J, Jorde R. Testosterone treatment improves body composition and sexual function in men with COPD, in a 6-month randomized controlled trial. Respir Med. 2004;98(9):906-913. doi:10.1016/j.rmed.2004.02.015

24. Köseoğlu N, Köseoğlu H, Ceylan E, Çimrin HA, Özalevli S, Esen A. Erectile dysfunction prevalence and sexual function status in patients with chronic obstructive pulmonary disease. J Urol. 2005;174(1):249-252. doi:10.1097/01.ju.0000163259.33846.74

25. Karadag F, Ozcan H, Karul AB, Ceylan E, Cildag O. Correlates of erectile dysfunction in moderate-to-severe chronic obstructive pulmonary disease patients. Respirology. 2007;12(2):248-253. doi:10.1111/j.1440-1843.2006.01042.x

26. Kaptein AA, van Klink RCJ, de Kok F, et al. Sexuality in patients with asthma and COPD. Respir Med. 2008;102(2):198-204. doi:10.1016/j. rmed.2007.09.012

27. Thomsen D, Jensen BO. Patients' experiences of everyday life after lung transplantation. J Clin Nurs. 2009;18(24):3472-3479. doi:10.1111/j.13652702.2009.02828.x

28. Collins EG, Halabi S, Langston M, Schnell T, Tobin MJ, Laghi F. Sexual dysfunction in men with COPD: impact on quality of life and survival. Lung. 2012;190(5):545-556. doi:10.1007/s00408-012-9398-4

29. Hansen MF, Willemoes J, Nielsen SL, Kristensen E, Giraldi A, Hansen EF. Sexual dysfunction, depression and well-being among patients with COPD or heart failure. Am J Respir Crit Care Med. 2012;185:A3343.

30. Uzaslan E, Mahboub B, Beji M, et al. The burden of chronic obstructive pulmonary disease in the Middle East and North Africa: results of the BREATHE study. Respir Med. 2012;106:S45-S59. doi:10.1016/S0954-6111(12)70014-8

31. Kahraman H, Sen B, Koksal N, Kilinc M, Resim S. Erectile dysfunction and sex hormone changes in chronic obstructive pulmonary disease patients. Multidiscip Respir Med. 2013;8(1):66. doi:10.1186/2049-6958-8-66

32. Mulhall AM, Lach LA, Krzywkowski-Mohn SM, Welge JA, Panos RJ. Therapeutic paralysis in veterans with COPD. Respir Med. 2013;107 (10):1547-1557. doi:10.1016/j.rmed.2013.05.013

33. Panos RJ, Krywkowski-Mohn SM, Sherman SN, Lach LA. Patient reported determinants of health: a qualitative analysis of veterans with chronic obstructive pulmonary disease. Chronic Obstr Pulm Dis. 2013;10(3):333-347. doi:10.3109/15412555.2012.752805

34. Theander K, Hasselgren M, Luhr K, Eckerblad J, Unosson M, Karlsson I. Symptoms and impact of symptoms on function and health in patients with chronic obstructive pulmonary disease and chronic heart failure in primary health care. Int J COPD. 2014;9:785-794. doi:10.2147/COPD. S62563

35. Abd-Elsalam FG, Mahgoub NA, Ghoneim AH, Abou-Abdu SE. Chronic obstructive pulmonary disease and sexual functioning among women in Egypt. Egypt J Chest Dis Tuberc. 2015;64(3):551-556. doi:10.1016/j.ejcdt.2015.02.006

36. Marques A, Jacome C, Cruz J, et al. Family-based psychosocial support and education as part of pulmonary rehabilitation in COPD: a randomized controlled trial. Chest. 2015;147(3):662-672. doi:10.1378/chest.14-1488

37. Shen TC, Chen WC, Lin CL, et al. The risk of erectile dysfunction in chronic obstructive pulmonary disease. Medicine. 2015;94(14):e448. doi:10.1097/MD.0000000000000448

38. Lauretti S, Cardaci V, Barrese F, Calzetta L. Chronic obstructive pulmonary disease (COPD) and erectile dysfunction (ED): results of the BRED observational study. Arch Ital Di Urol Androl. 2016;88(3):165-170.

39. Turan O, Ure I, Turan PA. Erectile dysfunction in COPD patients. Chron Respir Dis. 2016;13(1):5-12. doi:10.1177/1479972315619382

40. Dias M, Oliveira MJ, Oliveira P, Ladeira I, Lima R, Guimaraes M. Does any association exist between chronic obstructive pulmonary disease and erectile dysfunction? The DECODED study. Rev Port Pneumol. 2017;23(5):259-265. doi:10.1016/j.rppnen.2017.04.005

41. Hasan HM, Afify EI, Tawfik TM, Wakeel IMA, Fattah FMAE. Erectile dysfunction in male patients with chronic obstructive pulmonary disease. Al-Azhar Assiut Med J. 2017;15:67-70. doi:10.4103/AZMJ.AZMJ 5716

42. Anjum HMA, Akhtar N, Rashid H, Ahmad H. Frequency of erectile dysfunction in COPD patients. J Med Physiol Biophys. 2018;43:13-19.

43. Sinoj TM, Manu MK, Alex J, Mohapatra AK, Kamath A. Marital quality and erectile dysfunction among COPD: a cross-sectional study. $J$ Clin Diagnostic Res. 2018;12(9):OC01-OC04. 
44. Kawshty H, Makki M, Elmorsy W, Shabaan M, Ahmad A. Is chronic obstructive pulmonary disease a risk factor for erectile dysfunction? A crosssectional, comparative study. Al-Azhar Assiut Med J. 2019;17(1):79-85. doi:10.4103/AZMJ.AZMJ_26_19

45. Oh EG, Yoo JY. Progression of erectile function in men with chronic obstructive pulmonary disease: a cohort study. BMC Pulm Med. 2019;19:139. doi:10.1186/s12890-019-0902-y

46. Macêdo TE, da Silva G, Morano MT, Holanda MA, Pereira ED. Sexuality and associated factors in chronic obstructive pulmonary disease (COPD) patients attending a referral hospital in Northeastern Brazil. J Sex Marital Ther. 2020. doi:10.1080/0092623X.2020.1869124

47. Zysman M, Rubenstein J, Le Guillou F, et al. COPD burden on sexual well-being. Respir Res. 2020;21(1):1-10. doi:10.1186/s12931-020-01572-0

48. Kamal EE, Zedan HM, Ismail AA, Hussein K, Zakaria M, Badran AY. Erectile dysfunction in Egyptian patients with chronic obstructive pulmonary disease. Egypt J Dermatol Venerol. 2020;41:38-44.

49. Rosen RC, Cappelleri JC, Smith MD, Lipsky J, Peñ BM. Development and evaluation of an abridged, 5-item version of the international index of erectile function (IIEF-5) as a diagnostic tool for erectile dysfunction. Int J Impot Res. 1999;11(6):319-326. doi:10.1038/sj.ijir.3900472

50. Rosen RC, Riley A, Wagner G, Osterloh IH, Kirkpatrick J, Mishra A. The international index of erectile function (IIEF): a multidimensional scale for assessment of erectile dysfunction. Urology. 1997;49(6):822-830. doi:10.1016/S0090-4295(97)00238-0

51. Karacan I, Salis PJ, Thornby JI, Williams RL. The ontogeny of nocturnal penile tumescence. Waking Sleep. 1979;1:21-44.

52. Karacan I, Salis PJ, Ware C. Nocturnal penile tumescence and diagnosis in diabetic impotence. Am J Psychiatry. 1978;135:191-197.

53. Fisher C, Gross J, Zuch J. Cycle of penile erections synchronous with dreaming (REM) sleep. Arch Gen Psychiatry. 1965;12:29. doi:10.1001/ archpsyc.1965.01720310031005

54. Eekhof JAH, van Selm JH, Tombrock CFJL, Hoogslag GFW, Kaptein AA. De seksualiteitsbeleving van oudere patiënten met COPD [Sexual experiences of elderly patients with COPD]. Huisarts Wet. 1991;34:527-530.

55. Abdo CHN. The male sexual quotient: a brief, self-administered questionnaire to assess male sexual satisfaction. J Sex Med. 2007;4(2):382-389. doi:10.1111/j.1743-6109.2006.00414.x

56. Abdo CHN. Development and validation of female sexual quotient - a questionnaire to assess female sexual function. Rev Bras Med. 2006;63 (9):477-482

57. Laumann EO, Paik A, Glasser DB, et al. A cross-national study of subjective sexual well-being among older women and men: findings from the global study of sexual attitudes and behaviors. Arch Sex Behav. 2006;35(2):145-161. doi:10.1007/s10508-005-9005-3

58. Mcgahuey CA, Gelenberg AJ, Laukes CA, et al. The Arizona sexual experience scale (ASEX): reliability and validity. J Sex Marital Ther. $2000 ; 26$ (1):25-40. doi:10.1080/009262300278623

59. Rosen R, Brown C, Heiman J, et al. The female sexual function index (FSFI): a multidimensional self-report instrument for the assessment of female sexual function. J Sex Marital Ther. 2000;26(2):191-205. doi:10.1080/009262300278597

60. Portenoy RK, Thaler HT, Kornblith AB, et al. The memorial symptom assessment scale: an instrument for the evaluation of symptom prevalence, characteristics and distress. Eur J Cancer. 1994;30A(9):1326-1336. doi:10.1016/0959-8049(94)90182-1

61. Vennix P. NISSO-schalen: vragenlijsten voor de man en voor de vrouw [NISSO-scales: questionnaires for the man and for the woman]. Zeist, The Netherlands: NISSO, Netherlands Institute for Social Sexuality Research; 1985.

62. Derogatis LR. The psychosocial adjustment to illness scale (PAIS). J Psychosom Res. 1986;30(1):77-91. doi:10.1016/0022-3999(86)90069-3

63. Luo L, Zhao S, Wang J, et al. Association between chronic obstructive pulmonary disease and risk of erectile dysfunction: a systematic review and meta-analysis. Int J Impot Res. 2020;32(2):159-166. doi:10.1038/s41443-019-0165-4

64. Allen MS, Walter EE. Erectile dysfunction: an umbrella review of meta-analyses of risk-factors, treatment, and prevalence outcomes. $J$ Sex Med. 2019;16:531-541. doi:10.1016/j.jsxm.2019.01.314

65. Laurent SM, Simons AD. Sexual dysfunction in depression and anxiety: conceptualizing sexual dysfunction as part of an internalizing dimension. Clin Psychol Rev. 2009;29(7):573-585. doi:10.1016/j.cpr.2009.06.007

66. Badr H, Acitelli LK. Re-thinking dyadic coping in the context of chronic illness. Curr Opin Psychol. 2017;13:44-48. doi:10.1016/j. copsyc.2016.03.001

67. Traa MJ, De Vries J, Bodenmann G, Den Oudsten BL. Dyadic coping and relationship functioning in couples coping with cancer: a systematic review. Br J Health Psychol. 2015;20:85-114. doi:10.1111/bjhp.12094

68. Meier C, Bodenmann G, Moergeli H, et al. Dyadic coping among couples with COPD: a pilot study. J Clin Psychol Med Settings. 2012;19:243254. doi:10.1007/s10880-011-9279-7

69. Stenzel NM, Vaske I, Kühl K, Kenn K, Rief W. Prediction of end-of-life fears in COPD - hoping for the best but preparing for the worst. Psychol Health. 2015;30(9):1-18.

70. Siltanen H, Aine T, Huhtala H, Kaunonen M, Paavilainen E. The information needs of people with COPD-The holistic approach with special reference to gender and time since diagnosis. Nurs Open. 2021;8(5):2498-2508. doi:10.1002/nop2.773

71. Mercer B. Interviewing people with chronic illness about sexuality: an adaptation of the PLISSIT model. J Clin Nurs. 2008;17:341-351. doi:10.1111/j.1365-2702.2008.02582.x

72. Rubio-Rask SE, Farver-Vestergaard I, Hilberg O, Løkke A. Sexual health communication in COPD: the role, contents and design of patient information leaflets. Chron Respir Dis. 2021;18:147997312110203. doi:10.1177/14799731211020322

73. Marinelli L, Lanfranco F, Motta G, Zavattaro M. Erectile dysfunction in men with chronic obstructive pulmonary disease. $J$ Clin Med. 2021;10:2730. doi:10.3390/jcm10122730

74. Liberati A, Altman D, Tetzlaff J, et al. The PRISMA statement for reporting systematic reviews and meta-analyses of studies that evaluate health care interventions: explanation and elaboration. Journal of Clinical Epidemiology. 2009;62(10):e1-e34. 
International Journal of Chronic Obstructive Pulmonary Disease

Dovepress

\section{Publish your work in this journal}

The International Journal of COPD is an international, peer-reviewed journal of therapeutics and pharmacology focusing on concise rapid reporting of clinical studies and reviews in COPD. Special focus is given to the pathophysiological processes underlying the disease, intervention programs, patient focused education, and self management protocols. This journal is indexed on PubMed Central, MedLine and CAS. The manuscript management system is completely online and includes a very quick and fair peer-review system, which is all easy to use. Visit http://www. dovepress.com/testimonials.php to read real quotes from published authors.

Submit your manuscript here: https://www.dovepress.com/international-journal-of-chronic-obstructive-pulmonary-disease-journal 